
\title{
28 Research Suare \\ Tumor Cells Talk to Normal Cells Through Exosomes to Rebuild the Tumor Microenvironment
}

\section{Chunwen $\mathrm{Pu}$}

Sixth People's Hospital of Dalian

Qi Wang

Sixth People's Hospital of Dalian

\section{Aijun Sun}

Sixth People's Hospital of Dalian

\section{Ping Sun}

Sixth People's Hospital of Dalian

Hui Huang

Sixth People's Hospital of Dalian

Qiqi Zhang

Sixth People's Hospital of Dalian

\section{Xueying Sun}

Sixth People's Hospital of Dalian

\section{ZhiDong Wang ( $\nabla$ pucw@Inhc.net.cn )}

Sixth Peoples Hospital of Dalian https://orcid.org/0000-0003-0577-8826

\section{Yong Zhang}

Sixth People's Hospital of Dalian

\section{Primary research}

Keywords: Exosomes, liver cancer,GPC3,tumor microenvironment, Wnt / $\beta$-catenin signaling pathway

Posted Date: May 4th, 2021

DOl: https://doi.org/10.21203/rs.3.rs-444267/v1

License: (c) (i) This work is licensed under a Creative Commons Attribution 4.0 International License. Read Full License 


\section{Abstract}

\section{Background}

Exosomes play a key role in the growth of normal cells and various diseases such as cancer. Tumor exosomes regulate the connection between normal cells and cancer cells in the tumor microenvironment, thereby promoting the growth and invasion of cancer cells.

\section{Methods}

We used HepG2 cells silenced by shRNA targeting GPC3, LO2 and HepG2 cells treated with different concentrations of GPC3. We determined the effects of GPC3 on cell proliferation, apoptosis and invasion using CCK8, flow cytometry and Transwell, and Western blotting Method to determine the expression of GPC3/WNT3A/ß-catenin.HepG2 exosomes (Exo) and HepG2 exosomes treated with shRNA targeting GPC3 (sh-GPC3-Exo) were used to treat LO2 and HepG2 cells separately. Cell proliferation was measured by CCK8 experiment.The cell cycle and apoptosis were measured by flow cytometry. The cell invasion ability was analyzed by Transwell. The expression of GPC3/WNT3A/ $\beta$-catenin signal protein was determined by Western blotting.

Results

This is the first study to prove the bidirectional regulation of GPC3 between normal cells and liver cancer cells. After treatment of LO2 cells and HepG2 cells with GPC3, the LO2 cell cycle was blocked in the G0/G1 phase, while inhibiting cell proliferation, promoting cell apoptosis and invasion, but for HepG2 cells it appeared to promote proliferation.Silencing GPC3 can inhibit the proliferation and invasion, and promote cell apoptosis of HepG2. Subsequent experiments found that the expression of GPC3 was found in both LO2 and HepG2 exosomes, and the expression of GPC3 in HepG2 exosomes was significantly higher than that in LO2 exosomes. These suggest that GPC3 in exosomes has the potential to become a biomarker of HCC.In addition, HepG2 exosomes (Exo) can inhibit the proliferation of LO2 cells and promote apoptosis and invasion, which is consistent with the effect of GPC3 treatment. We also found that GPC3 is contained in HepG2 exosomes (shGPC3-Exo) that have silenced GPC3, which has the same effect on LO2 cells as HepG2 exosomes (Exo), but the degree of influence is reduced. shGPC3-Exo showed a promoting effect on the proliferation of HepG2 cells, but inhibited cell invasion. Therefore, GPC3 in Exo plays a role in the proliferation of LO2 cells and HepG2 cells. Further studies have shown that GPC3 in liver cancer exosomes regulates the proliferation, apoptosis and invasion of LO2 and HepG2 cells through the Wnt / $\beta$-catenin signaling pathway.

\section{Conclusion}

GPC3 in the exosomes of liver cancer cells inhibits the proliferation of normal liver cells and promotes apoptosis by activating the Wnt/ $\beta$-catenin signaling pathway, promotes the proliferation of liver cancer cells, and assists the occurrence and development of HCC. 


\section{Introduction}

The incidence of liver cancer is the fifth highest among cancers in the world. There will be 905,700 new cases worldwide in 2020 , with a mortality rate as high as $90 \%{ }^{[1]}$. Hepatocellular carcinoma (HCC) is the most common liver malignant tumor, accounting for about $90 \%$ of all cases ${ }^{[2]}$. The treatment of liver cancer patients is very complicated. Traditional surgical resection is the main choice for patients. However, $\mathrm{HCC}$ is prone to metastasis and recurrence. Because HCC patients are usually diagnosed at an advanced stage, their survival rate is still very low $^{[3]}$. Early diagnosis and treatment are the key to improving the survival time of patients with liver cancer ${ }^{[4]}$. Therefore, the identification of new liver cancer diagnostic markers is very important.

Exosomes are membranous vesicles released into the cell matrix after fusion of intracellular multivesicular bodies and cell membranes. Exosomes in tumors carry specific markers derived from tumor cells, and their contents are related to the invasion ability of tumor cells and the tumor microenvironment ${ }^{[5,6]}$. More and more studies have shown that exosomes play a key role in the growth of normal cells and various diseases including cancer. Tumor cell-derived exosomes not only participate in cell-to-cell communication, but also affect the continuous growth, invasion and migration of tumor cells $^{[7]}$. Similarly, tumor exosomes can be taken up by other tumor cells, thereby causing cancer cells to metastasize and spread ${ }^{[8]}$. Exosomes in cancer patients can be used as a type of biopsy, and exosomal proteins are used as new biomarkers to provide new opportunities for early diagnosis of cancer ${ }^{[9-11]}$.

The heparin sulfate proteoglycan glypican-3 (GPC3) has the ability to regulate cell proliferation, adhesion and migration ${ }^{[12,13]}$. In addition, the expression of GPC3 is elevated in more than $70 \%$ of hepatocellular carcinoma $(\mathrm{HCC})^{[14]}$, which is related to the invasion and metastasis of liver cancer. GPC3 also promotes HCC growth by stimulating the classic wnt/ $\beta$-catenin signaling pathway, while GPC3 gene silencing inhibits HCC cell proliferation and induces apoptosis ${ }^{[15]}$. Pei et al. found that autophagy inhibits the proliferation of HepG2 liver cancer cells by inhibiting GPC3/wnt/ $\beta$-catenin signal transduction ${ }^{[16]}$. In addition, the expression of GPC3 is related to the poor prognosis of HCC patients ${ }^{[17]}$. Therefore, GPC3 is a suitable biomarker and prognostic factor for $\mathrm{HCC}$, which can be used as a biomarker for early diagnosis of hepatocellular carcinoma, and targeted inhibition of GPC3 signaling may help control the proliferation and metastasis of HCC cells ${ }^{[18]}$. The role of GPC3 in liver cancer is still controversial, and its exact mechanism and clinical significance are still inconclusive.

Previous studies have shown that the expression of GPC3 in serum exosomes of HCC patients is significantly higher than that of hepatitis $B$ patients and healthy people ${ }^{[19]}$. This study explored the effect of GPC3 in HCC exosomes on the proliferation and invasion of HCC cells and on related signal pathways. And we explored the possible effect of GPC3 in tumor exosomes on normal liver cells and the impact of tumor microenvironment, which provides a theoretical basis for studying the occurrence and development of HCC. 


\section{Methods}

Cell culture

All cells were purchased from Type Culture Collection of the Chinese Academy of Sciences (Shanghai, China). HepG2 was cultured and passaged in MEM supplemented with $0.4 \mathrm{mg} / \mathrm{ml} \mathrm{G} 418$ and $10 \%$ FBS. LO2 was cultured and passaged in RPMI- 1640 supplemented with $1 \% \mathrm{P} / \mathrm{S}$ and $10 \% \mathrm{FBS}$ at $37^{\circ} \mathrm{C}$ and $5 \%$ C02. GPC3 was from CUSABIO (Wuhan, China).

\section{Isolation of exosomes from medium}

The collected medium was centrifuged at $300 \mathrm{~g}$ at 4 degrees for 10 minutes to remove cell pellets. Then, the supernatant was centrifuged at $2000 \mathrm{~g}$ for 4 minutes and 10 minutes to remove dead cells. Then, the supernatant was centrifuged at 10,000 g at 4 degrees for 30 minutes to remove cell debris. Finally, the supernatant was transferred to an ultracentrifugation adapter tube, and centrifuged at $100,000 \mathrm{~g}$ for 70 min at $4^{\circ} \mathrm{C}$ to obtain a precipitate, which was an isolated exosomes. After adding $30 \mathrm{~mL}$ of $1 \times \mathrm{PBS}$ to the pellet, resuspend and mix the mixture, and centrifuge at $100,000 \mathrm{~g}$ for $70 \mathrm{~min}$. Discard the supernatant, add $250 \mu \mathrm{L}$ of $1 \times \mathrm{PBS}$ to the pellet, resuspend and mix, and store at $-80^{\circ} \mathrm{C}$ until use. ZetaViewPMX 110 (Particle Metrix, Meerbusch, Germany) and the corresponding software ZetaView8.04.02 were used to perform Nanoparticle Tracking Analysis (NTA) in Shanghai VivaCell to measure the particle size of exosomes.

\section{The enzyme-linked immunosorbent assay (ELISA) for GPC3}

According to the manufacturer's instructions, use CUSABIO's Human Glypican-3, GPC-3 ELISA Kit to measure the level of GPC3. This determination is performed in triplicate.

\section{Lentiviral, plasmid and cell transfection}

The genOFFTM st-h-GPC3 (CGACACCCTTTGCTGGAAT) designed by Siwega (Wuhan, China) for GPC3 was transfected with $90 \%$ confluent HepG2 cells with lentivirus (hU6-MCS-CBh-gcGFP-IRES-puromycin GV493). After 3 days of infection, observe the expression of the reporter gene GFP on the lentivirus. If the infection efficiency is greater than $50 \%$, continue the culture. After the infection time reaches 5 days, the cells are extracted and RNA is collected for PCR detection.

\section{Quantitative real-time PCR}

Use TRIzol to separate cellular and exosomal RNA. Use iScript cDNA Synthesis Kit (Bio-Rad, USA) to synthesize first-strand cDNA with random primers. QPCR was performed on the CFX96 real-time PCR detection system (Bio-Rad, USA) using iTaqTM universal SYBR Green Supermix (Bio-Rad, USA). The mRNA content is normalized to the housekeeping gene GAPDH. Table I summarizes the primer sequences for all RT qPCR. The reaction conditions are: $95^{\circ} \mathrm{C}$ for 5 minutes, then use the CFX Connect system at 95 
${ }^{\circ} \mathrm{C}$ for 10 seconds and $60^{\circ} \mathrm{C}$ for 30 seconds.According to the $2-\Delta \Delta \mathrm{Cq}$ method, the RT-qPCR results were normalized to GADPH.

Table 1. qPCR primer sequence

\begin{tabular}{|c|c|}
\hline Primer & Sequence $\mathbb{5} 5^{\prime}$ to $3^{\prime} \rrbracket$ \\
\hline Hu-GAPDH -F & ACAACTTTGGTATCGTGGAAGG \\
\hline Hu-GAPDH -R & GCCATCACGCCACAGTTTC \\
\hline Hu-GPC3-F & GAAACAGTCAGCAGGCAAC \\
\hline Hu-GPC3-R & GAAGCACACCACCGAGA \\
\hline
\end{tabular}

\section{CCK-8 kit to evaluate cell proliferation}

LO2 cells, HepG2 and HepG2-shGPC3 cells were seeded into 96-well plates at a density of 3-5×104 cells/well, placed in $100 \mu \mathrm{l}$ medium, and then treated with various concentrations of drugs. After incubating at $37^{\circ} \mathrm{C}$ for 48 hours, add $10 \mu \mathrm{L}$ of CCK-8 solution to each well of the culture medium and mix well to ensure the color uniformity in the well. After continuing to incubate for $4 \mathrm{~h}$, the absorbance was measured directly at $450 \mathrm{~nm}$. The percentage of cell proliferation for each treatment was calculated as (OD value of drug-added group/OD value of control group) $\times 100 \%$.

\section{Cell invasion assay}

The cell invasion was measured by the Transwell assay. The cells $\left(10^{5}\right.$ cells/well) were divided into different groups and seeded in a serum-free medium with Matrigel gel in the upper chamber. At the same time, the lower chamber is filled with $500 \mu \mathrm{L}$ of the corresponding medium containing $20 \%$ FBS, and cultured in the incubator for $24 \mathrm{~h}$. Take out the Transwell chamber, discard the culture medium in the well, wash twice with calcium-free PBS, fix with $4 \%$ paraformaldehyde for 10 minutes; wash twice with PBS, 2 minutes each time; stain with crystal violet for 10 minutes, wipe gently with a cotton swab Remove the upper layer of Matrigel and cells and wash them with PBS 3 times; randomly select five fields of view to observe the cells under a 200x microscope, and use Inversion Microscope (OLYMPUS) for imaging.

\section{Flow cytometry analysis of cell apoptosis and cell cycle}

The cells were seeded in a 6-well plate at a concentration of $1 \times 10^{5} /$ well and treated with GPC3 $(10 \mu \mathrm{M})$ or HepG2 exosomes $(5 \mu \mathrm{M})$ or HepG2 exosomes knocked down GPC3 (50 ng/ $\mu \mathrm{L}) .24$ hours after treatment, according to the manufacturer's instructions, use Annexin V / PI detection kit (BD Biosciences) and cell cycle staining kit (MultiSciences) to detect apoptosis and cell cycle, and then analyze on BDAccuriß.

\section{Western blotting measures protein expression in cells and exosomes}


According to our previous article ${ }^{[30]}$, Western blot analysis was performed to detect CD63 (1:1000, abcam), TSG101 (1:1000, abcam), GPC3 (1:1000, bioss), WNT3A (1:1000, HuaBio ), $\beta$-Catenin (1:1000, HuaBio) and GAPDH (1:10000, abcam) protein level report.

\section{Statistical analysis}

Results were expressed as the mean $\pm S E M$. To calculate statistical significance, the student's $t$ test (twotailed) (when comparing two groups) or one-way ANOVA followed by Tukey's multiple comparisons test (when comparing three or more groups) were used. $P$ value $<0.05$ were considered significant. All calculations were performed using Prism software.

\section{Results}

\section{Exosomes isolation and verification}

Previous experimental results found that the expression level of GPC3 in liver cancer tissues was significantly higher than that of normal controls. In order to prove the expression of GPC3 in HCC cell lines, we selected normal liver cells LO2 and representative HCC cells HepG2.

Nanoparticle tracking analysis (NTA) was performed with ZetaViewPMX 110 to observe the particle size of exosomes in the cell culture medium. The sample showed a vesicle structure with a diameter of about 50-150 nm, which was in line with the morphological characteristics of exosomes in the literature ${ }^{[20]}$

(Figure 1A). Western blot analysis showed the presence of CD63 and TSG101, which were reported to be exosomal markers ${ }^{[21]}$ (Figure 1B). The results indicate that exosomes have been successfully isolated from LO2 and HepG2 cell lines. In this study, we verified the expression of GPC3 in exosomes of LO2 and HepG2 cell lines by Western blot and ELISA. The expression of GPC3 in liver cancer cell line HepG2 exosomes was more than that in normal liver cell line LO2 exosomes, which was consistent with the comparison of GPC3 expression in the two cells (Figure 1C,D).

\section{The effect of GPC3 on the proliferation and apoptosis of normal cells}

Studies have shown that GPC3 promotes the development of liver cancer, but due to the low content of GPC3 in healthy human tissues, there is no research on the effect of GPC3 on normal cells. In order to explore whether the effect of GPC3 on the proliferation and invasion of normal liver cells is different from that of liver cancer cells, we added GPC3 to LO2 and HepG2 cells to study the effects of different concentrations of GPC3 on cell proliferation. After adding different concentrations of GPC3, the growth of LO2 cells was inhibited, and the growth of HepG2 cells was promoted. The effect of GPC3 increased first

and then decreased with the increase of concentration. GPC3 at a dose of $1 \mathrm{mg} / \mathrm{ml}$ had the most obvious effect on the two types of cells (Figure 2A). Flow cytometry analysis of Annexin V/PI staining also showed that apoptotic cells increased in LO2 cells after the addition of GPC3 at a concentration of 1 $\mathrm{mg} / \mathrm{ml}$, while there was no difference in the level of apoptosis between GPC3 treated and untreated HepG2 cells (Figure 2B). Subsequently, we tested whether GPC3 affects cell growth through cell cycle 
arrest. As shown in Figure 2C, the addition of GPC3 resulted in an increase in the number percentage of the G0/G1 population of LO2 cells, resulting in G0/G1 block; on the contrary, the number percentage of the G0/G1 population decreased in HepG2 cells. Later, in the transwell analysis of cell invasion ability, the invasion ability of LO2 cells and HepG2 cells increased after adding GPC3 (Figure 2D). These data indicated that GPC3 can inhibit the proliferation of normal cells by enhancing cell apoptosis and cell cycle arrest, while for HCC cells it can reduce cell cycle arrest to promote the proliferation of HCC cells, and not effect on cell apoptosis. GPC3 has the ability to promote the invasion of normal liver cells and HCC cells.

In order to further explore the role of GPC3 in the growth of HCC cells, shRNA targeting GPC3 was transfected into HepG2 cells. After transfection, the expression of GPC3 in HepG2 cells decreased in mRNA and protein levels (Figure 3A-C). The growth of HepG2 cells was inhibited after knockdown (Figure 3D). Flow cytometry analysis of Annexin V/PI staining also showed an increase in apoptotic cells in HepG2 cells after GPC3 knockdown (Figure 3E). Subsequently, we tested whether cell cycle arrest affected the growth of GPC3 knockdown HepG2 cells. As shown in Figure 3F, knockdown of GPC3 led to an increase in the percentage of the G0/G1 population of HepG2 cells, showing a G0/G1 blocking trend. Next, transwell analysis was performed to evaluate the effect of GPC3 on HCC cell invasion. The results showed that the invasive ability of the HCC cell line knocked down by GPC3 was reduced (Figure 3G). These data indicated that GPC3 gene silencing in HCC cells can promote cell apoptosis and cell cycle arrest, thereby inhibiting the proliferation of HCC cells and inhibiting the invasion of HCC cells.

\section{Exosomal GPC3 stimulated the proliferation and migration of HCC cells}

The previous research found that GPC3 protein was detected in serum exosomes of HCC patients ${ }^{[19]}$, which was consistent with the expression of GPC3 in HCC tissues. Next, we will observe the effect of GPC3 in liver cancer exosomes on normal liver cells. LO2 and HepG2 cells were treated with HepG2 exosomes (Exo) and GPC3 knockdown HepG2 exosomes (shGPC3-Exo), respectively. The GPC3 content in exosomes of HepG2 cells decreased after GPC3 was knocked down (Figure 4A).

After adding Exo, the growth of LO2 cells was significantly inhibited, which was concentration-dependent (Figure 4B). shGPC3-Exo has the same inhibitory effect on the proliferation of LO2 cells, but the inhibitory effect is weakened, compared with the addition of Exo. shGPC3-Exo also promoted the growth of HepG2 cells in a dose-dependent manner (Figure 4B). This indicated that GPC3 in HCC exosomes had an inhibitory effect on the growth of normal hepatocytes.

Flow cytometry analysis of Annexin V/PI staining showed that after treatment with $100 \mu \mathrm{g} / \mathrm{mL}$ Exo or shGPC3-Exo, the number of apoptosis of LO2 cells increased, and the effect of Exo group was more obvious than that of shGPC3-Exo group. The addition of shGPC3-Exo to HepG2 cells did not affect the number of apoptosis (Figure 4C). Later, in the detection of the effect of exosomes on the liver cell cycle, it was found that the percentage of the number of LO2 cells G0/G1 population increased after the addition of the two groups of exosomes, showing G0/G1 blockade. The percentage of the number of HepG2 cells 
in the G0/G1 population decreased after shGPC3-Exo was added (Figure 4D). This indicated that GPC3 in HepG2 cell exosomes can promote the apoptosis of LO2 cells, and may promote apoptosis by inhibiting the cell cycle of LO2 cells.

Then we used Transwell experiment to analyze the influence of liver cancer exosomes on the migration ability of normal liver cells and HCC cells. After adding Exo, the number of migrating cells in LO2 cells remained unchanged, while the number of migrating cells increased significantly after adding shGPC3Exo. shGPC3-Exo significantly reduced the number of HepG2 cell migration. The results showed that the migration effect of HepG2 exosomes on normal cells was related to the dose of GPC3 (Figure 4E).

These data indicate that HCC cells can promote the apoptosis of normal hepatocytes, inhibit cell cycle and cell proliferation, and affect the invasion of normal hepatocytes through GPC3 in their exosomes; while GPC3 in HCC exosomes can increase cell cycle by promoting cell cycle. Proliferation of HCC cells.

\section{GPC3 in liver cancer exosomes affects Wnt/ $\beta$-catenin signaling pathway in HCC cells}

Wnt/ $\beta$-catenin signaling pathway plays an important role in promoting the proliferation and migration of HepG2 cells, and plays an important role in the occurrence, development and metastasis of liver cancer. It is an important molecular mechanism of liver cancer. Studies have shown that in liver cancer, GPC3 plays a role through the $\mathrm{Wnt} / \beta$-catenin signaling pathway ${ }^{[22]}$. The above experiments also showed that GPC3 in liver cancer exosomes affected the proliferation of normal liver cells.In the follow-up, we will investigate whether GPC3 in liver cancer exosomes also affects the proliferation of normal liver cells through the Wnt/ $\beta$-catenin signaling pathway.

First, compare the GPC3 and Wnt/ $\beta$-catenin signaling pathways in hepatocytes and hepatocyte exosomes. Western blot results showed that the GPC3 content of LO2 cell exosomes was significantly less than that in the cells, and the expression of Wnt3a and $\beta$-catenin protein was uniformly reduced; and the GPC3, Wnt3a and $\beta$-catenin protein content of HepG2 exosomes were also less than that of HepG2 The content in cells (Figure 5A, B).

Later, it was found in further studies that the GPC3 content in LO2 cells increased significantly after GPC3 treatment, while Wnt3a and $\beta$-catenin protein increased; after Exo was added, the GPC3, Wnt3a and $\beta$ catenin protein content in LO2 cells also increased significantly. After shGPC3-Exo, the contents of GPC3, Wnt3a and $\beta$-catenin were increased compared with the control group LO2 cells, but the protein content was lower than that of Exo (Figure 5C, D). It showed that GPC3 in HepG2 cell exosomes increased GPC3, Wnt3a and $\beta$-catenin protein content in LO2 cells. In the study of HepG2 cells, it was found that after knocking down the GPC3 in the cells, as the GPC3 in the cells decreased significantly, the content of Wnt3a and $\beta$-catenin protein decreased;After adding GPC3 to HepG2 cell culture, it was found that the content of GPC3 and $\beta$-catenin protein increased significantly, and the content of Wnt3a also increased (Figure 5C, D). After adding shGPC3-Exo, the contents of GPC3, Wnt3a and $\beta$-catenin in HepG2 cells increased compared with the control group. 
The results of these studies indicate that the levels of GPC3 and Wnt/ $\beta$-catenin signaling protein in normal liver cells are significantly lower than those in liver cancer cells. GPC3 in liver cancer cell exosomes can activate Wnt/ $\beta$-catenin signaling pathway by increasing GPC3 in normal liver cells.

\section{Discussion}

The "Global Cancer Report 2020" published by the WHO pointed out that there are more than 900,000 liver cancer patients worldwide and 830,000 death $s^{[1]}$. The leading cause of death in patients with liver cancer is tumor progression and metastasis. The tumor microenvironment determines the type of liver cancer [23], because tumor-specific markers carried by tumor exosomes participate in cell communication in the tumor microenvironment, and are related to the growth and invasion ability of tumor cells. In recent years, researchers have found that the expression rate of Glypican-3 in liver cancer tissues has gradually increased significantly with the increase of clinical stages ${ }^{[24]}$. We also found increased levels of Glypican-3 in the blood of liver cancer patients.. Huixia Di et al. also found that compared with healthy people and hepatitis B patients, the expression of GPC3 in serum exosomes of HCC patients can be detected and the content is significantly increased ${ }^{[19]}$, indicating that liver cancer cells can secrete GPC3 into the blood circulation. We found in in vitro experiments that GPC3 was expressed in normal liver cells and HCC cells and their exosomes, and the GPC3 content in HCC cell exosomes was significantly higher than that in normal liver cell exosomes. It shows that when GPC3 promotes the metastasis of liver cancer cells, it may also carry out cell communication with normal liver cells, affecting the growth of normal liver cells, thereby helping the metastasis of tumor cells. Therefore, elucidating the role of GPC3 in hepatocytes and signal transduction pathways in exosomes in the future may provide a theoretical basis for revealing the development process of liver cancer and determining new early diagnosis methods.

Shanshan W et al. found that in the proliferation and migration of HCC cells, GPC3 showed a significant promoting effect ${ }^{[25]}$, which is consistent with our experimental results. In our research on HCC cell apoptosis, we found that the number of apoptosis of HCC cells remains unchanged after GPC3 is added, but the number of apoptosis of HCC cells increases after GPC3 gene knockdown. Therefore, GPC3 in HCC cells may inhibit cell apoptosis. This is the first time we have discovered that GPC3 inhibits the proliferation of normal hepatocytes by blocking the cell cycle and significantly promotes their apoptosis. Therefore, GPC3 may help HCC cell proliferation by inducing normal liver cell apoptosis and proliferation, and further promote the development of liver cancer. Studies have found that GPC3 stimulates the growth of $\mathrm{HCC}$ cells by increasing the autocrine/paracrine pathway and activating the canonical Wnt signaling pathway ${ }^{[15]}$. We found that for normal liver cells, GPC3 also activates the classic wnt / $\beta$-catenin signaling pathway, which further promotes the occurrence of HCC.

Exosomes transmit information through the contents of vesicles ${ }^{[26]}$. Exosomes secreted by tumor cells can regulate surrounding or distant non-tumor cells to form a pre-metastasis microenvironment ${ }^{[27,28]_{\text {to }}}$ promote tumor cell metastasis. We next tested whether GPC3 in liver cancer exosomes can perform its function in normal liver cells. The optimal concentration of liver cancer exosomes $(100 \mu \mathrm{g} / \mathrm{mL})$ to 
promote proliferation acts on liver cell lines. In normal liver cells, liver cancer exosomes can significantly inhibit proliferation, block cell cycle, and promote apoptosis. After the decrease of GPC3 content in liver cancer exosomes, the effect on normal cells is reduced, which proves that the exosomes of liver cancer GPC3 can inhibit the proliferation of normal liver cells and promote apoptosis. Liver cancer exosomes have no effect on the migration of normal liver cells, but the reduction of GPC3 content in liver cancer exosomes can significantly promote the migration of normal liver cells, which proves that the migration effect of liver cancer exosomes on normal cells is related to the dose of GPC3. The above results indicate that the content of GPC3 in liver cancer cell exosomes is significantly higher than that of normal liver cell exosomes, while GPC3 in liver cancer cell exosomes inhibits the proliferation of normal liver cells, promotes apoptosis, and can promote the proliferation of HCC cells, Which can contribute to the development of HCC.

The activation of the Wnt signaling pathway is one of the most common molecular events related to the progression of HCC. Further studies have shown that the Wnt signaling pathway is essential for GPC3-

mediated cell growth ${ }^{[29]}$. However, the regulatory mechanism of GPC3 in HCC exosomes involved in HCC cell growth is still unclear. In this study, we proved that GPC3 and wnt / $\beta$-catenin signaling protein exist in the exosomes of normal hepatocytes and HCC cells, and the content of wnt / $\beta$-catenin signaling protein is significantly lower than that in cells. GPC3 in the exosomes of HCC cells can activate the Wnt/ $\beta$-catenin pathway of normal hepatocytes, and the effect is reduced after adding HepG2-shGPC3-exo, which is consistent with the trend of adding GPC3.This further proves that GPC3 in HCC cell exosomes activates the Wnt / $\beta$-catenin pathway in normal liver cells, thereby promoting the occurrence of HCC.

\section{Conclusion}

This study shows that GPC3 is enriched and expressed in liver cancer exosomes. It not only regulates the cell cycle and migration of HCC, but also inhibits the proliferation and migration of normal hepatocytes, and activates the Wnt / $\beta$-catenin pathway in normal hepatocytes, thereby promoting the occurrence and development of HCC.

\section{Abbreviations}

GPC3,glypican-3; Exo,Exosomes;shGPC3-Exo,HepG2 exosomes that have silenced GPC3

\section{Declarations}

\section{Acknowledgements}

This study was supported by Natural Science Foundation Guidance Program Project of Liaoning Province (20180550784), Natural Science Foundation Guidance Program Project of Liaoning Province2019-ZD1003). 
ZDW, CWP and YZ proposed and guided this research. QW, HH, PS, QQZ and XYS were the experimental operators. QW and CWP collected and analyzed the data. QW, HH, PS, QQZ, XYS and AJS contributed to the design of the study and further drafts. ZDW, CWP and YZ revised the manuscript.All authors read and approved the final manuscript.

\section{Funding}

This research has received no funds.

\section{A vailability of data and materials}

The datasets used during the current study are available from the corresponding author on reasonable request.

\section{Ethics approval and consent to participate}

Not applicable.

\section{Consent for publication}

Not applicable.

\section{Competing Interests}

The authors have declared that no competing interest exists.

\section{References}

1. International Agency for Research on Cancer:Liver. World Health Organization (WHO) 2020.

2. Llovet JM, Zucman-Rossi J, Pikarsky E, Sangro B, Schwartz M, Sherman M, Gores G. Hepatocellular carcinoma. Nat Rev Dis Primers 2016; 2: 16018.

3. Cao H, Phan H, Yang LX. Improved chemotherapy for hepatocellular carcinoma. Anticancer Res 2012; 32(4): 1379-1386.

4. Kudo M. Emerging strategies for the management of hepatocellular carcinoma. Dig Dis 2014; 32(6): 655-657.

5. Meckes DG, Jr. Exosomal communication goes viral. J Virol 2015; 89(10): 5200-5203.

6. Tkach M, Thery C. Communication by Extracellular Vesicles: Where We Are and Where We Need to Go. Cell 2016; 164(6): 1226-1232.

7. Le MT, Hamar P, Guo C, Basar E, Perdigao-Henriques R, Balaj L, Lieberman J. miR-200-containing extracellular vesicles promote breast cancer cell metastasis. J Clin Invest 2014; 124(12): 5109-5128.

8. Costa-Silva B, Aiello NM, Ocean AJ, Singh S, Zhang H, Thakur BK, Becker A, Hoshino A, Mark MT, Molina H, Xiang J, Zhang T, Theilen TM, Garcia-Santos G, Williams C, Ararso Y, Huang Y, Rodrigues G, 
Shen TL, Labori KJ, Lothe IM, Kure EH, Hernandez J, Doussot A, Ebbesen SH, Grandgenett PM, Hollingsworth MA, Jain M, Mallya K, Batra SK, Jarnagin WR, Schwartz RE, Matei I, Peinado H, Stanger BZ, Bromberg J, Lyden D. Pancreatic cancer exosomes initiate pre-metastatic niche formation in the liver. Nat Cell Biol 2015; 17(6): 816-826.

9. Melo SA, Luecke LB, Kahlert C, Fernandez AF, Gammon ST, Kaye J, LeBleu VS, Mittendorf EA, Weitz J, Rahbari N, Reissfelder C, Pilarsky C, Fraga MF, Piwnica-Worms D, Kalluri R. Glypican-1 identifies cancer exosomes and detects early pancreatic cancer. Nature 2015; 523(7559): 177-182.

10. Corcoran C, Friel AM, Duffy MJ, Crown J, O'Driscoll L. Intracellular and extracellular microRNAs in breast cancer. Clin Chem 2011; 57(1): 18-32.

11. Skog J, Wurdinger T, van Rijn S, Meijer DH, Gainche L, Sena-Esteves M, Curry WT, Jr., Carter BS, Krichevsky AM, Breakefield XO. Glioblastoma microvesicles transport RNA and proteins that promote tumour growth and provide diagnostic biomarkers. Nat Cell Biol 2008; 10(12): 1470-1476.

12. Haruyama Y, Kataoka H. Glypican-3 is a prognostic factor and an immunotherapeutic target in hepatocellular carcinoma. World J Gastroenterol 2016; 22(1): 275-283.

13. Sung YK, Hwang SY, Park MK, Farooq M, Han IS, Bae HI, Kim JC, Kim M. Glypican-3 is overexpressed in human hepatocellular carcinoma. Cancer Sci 2003; 94(3): 259-262.

14. Baumhoer D, Tornillo L, Stadlmann S, Roncalli M, Diamantis EK, Terracciano LM. Glypican 3 expression in human nonneoplastic, preneoplastic, and neoplastic tissues: a tissue microarray analysis of 4,387 tissue samples. Am J Clin Pathol 2008; 129(6): 899-906.

15. Capurro MI, Xiang YY, Lobe C, Filmus J. Glypican-3 promotes the growth of hepatocellular carcinoma by stimulating canonical Wnt signaling. Cancer Res 2005; 65(14): 6245-6254.

16. Hu P, Cheng B, He Y, Wei Z, Wu D, Meng Z. Autophagy suppresses proliferation of HepG2 cells via inhibiting glypican-3/wnt/beta-catenin signaling. Onco Targets Ther 2018; 11: 193-200.

17. Shirakawa H, Suzuki H, Shimomura M, Kojima M, Gotohda N, Takahashi S, Nakagohri T, Konishi M, Kobayashi N, Kinoshita T, Nakatsura T. Glypican-3 expression is correlated with poor prognosis in hepatocellular carcinoma. Cancer Sci 2009; 100(8): 1403-1407.

18. Hippo Y, Watanabe K, Watanabe A, Midorikawa Y, Yamamoto S, Ihara S, Tokita S, Iwanari H, Ito Y, Nakano K, Nezu J, Tsunoda H, Yoshino T, Ohizumi I, Tsuchiya M, Ohnishi S, Makuuchi M, Hamakubo T, Kodama T, Aburatani H. Identification of soluble NH2-terminal fragment of glypican-3 as a serological marker for early-stage hepatocellular carcinoma. Cancer Res 2004; 64(7): 2418-2423.

19. Di H, Mi Z, Sun Y, Liu X, Li A, Jiang Y, Gao H, Rong P, Liu D. Nanozyme-assisted sensitive profiling of exosomal proteins for rapid cancer diagnosis. Theranostics 2020; 10(20): 9303-9314.

20. Yuan R, Zhi Q, Zhao H, Han Y, Gao L, Wang B, Kou Z, Guo Z, He S, Xue X, Hu H. Upregulated expression of miR-106a by DNA hypomethylation plays an oncogenic role in hepatocellular carcinoma. Tumour Biol 2015; 36(4): 3093-3100.

21. Baranyai T, Herczeg K, Onodi Z, Voszka I, Modos K, Marton N, Nagy G, Mager I, Wood MJ, El Andaloussi S, Palinkas Z, Kumar V, Nagy P, Kittel A, Buzas El, Ferdinandy P, Giricz Z. Isolation of 
Exosomes from Blood Plasma: Qualitative and Quantitative Comparison of Ultracentrifugation and Size Exclusion Chromatography Methods. PLoS One 2015; 10(12): e0145686.

22. Gao W, Ho M. The role of glypican-3 in regulating Wnt in hepatocellular carcinomas. Cancer Rep 2011; 1(1): 14-19.

23. Seehawer M, Heinzmann F, D'Artista L, Harbig J, Roux PF, Hoenicke L, Dang H, Klotz S, Robinson L, Dore G, Rozenblum N, Kang TW, Chawla R, Buch T, Vucur M, Roth M, Zuber J, Luedde T, Sipos B, Longerich T, Heikenwalder M, Wang XW, Bischof O, Zender L. Necroptosis microenvironment directs lineage commitment in liver cancer. Nature 2018; 562(7725): 69-75.

24. Sun B, Huang Z, Wang B, Yu Y, Lin S, Luo L, Wang Y. Significance of Glypican-3 (GPC3) Expression in Hepatocellular Cancer Diagnosis. Med Sci Monit 2017; 23: 850-855.

25. Wang S, Chen N, Chen Y, Sun L, Li L, Liu H. Elevated GPC3 level promotes cell proliferation in liver cancer. Oncol Lett 2018; 16(1): 970-976.

26. Gele AA, Qureshi SA, Kour P, Kumar B, Diaz E. Barriers and facilitators to cervical cancer screening among Pakistani and Somali immigrant women in Oslo: a qualitative study. Int J Womens Health 2017; 9: 487-496.

27. Peinado H, Alec kovic M, Lavotshkin S, Matei I, Costa-Silva B, Moreno-Bueno G, Hergueta-Redondo M, Williams C, Garcia-Santos G, Ghajar CM, Nitadori-Hoshino A, Hoffman C, Badal K, Garcia BA, Callahan MK, Yuan J, Martins VR, Skog J, Kaplan RN, Brady MS, Wolchok JD, Chapman PB, Kang Y, Bromberg J, Lyden D. Corrigendum: Melanoma exosomes educate bone marrow progenitor cells toward a pro-metastatic phenotype through MET. Nat Med 2016; 22(12): 1502.

28. Yu Z, Zhao S, Ren L, Wang L, Chen Z, Hoffman RM, Zhou J. Pancreatic cancer-derived exosomes promote tumor metastasis and liver pre-metastatic niche formation. Oncotarget 2017; 8(38): 6346163483.

29. Wu Y, Liu H, Ding H. GPC-3 in hepatocellular carcinoma: current perspectives. J Hepatocell Carcinoma 2016; 3: 63-67.

30. Huang H, Zhou Z, Li H, Zhang Y, Zhao L, Wang Z, Zhang Q, Liu C, Han C, Wang Q, Pu C, Zou W. Downregulation of ER-alpha36 mRNA in serum exosomes of the patients with hepatocellular carcinoma. Clin Transl Med 2020; 10(1): 346-352.

\section{Figures}


A

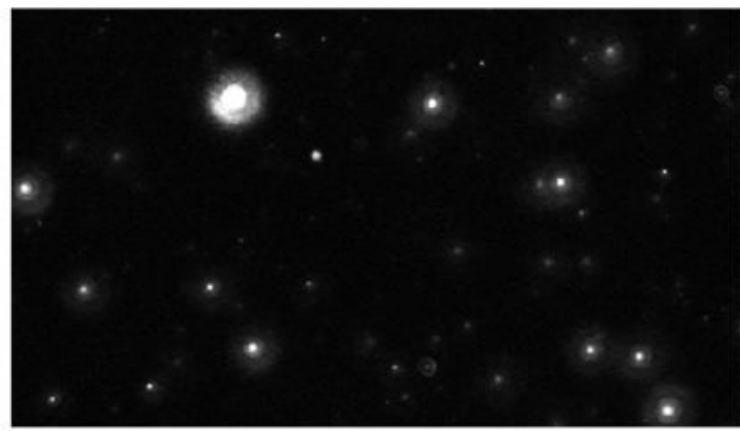

C

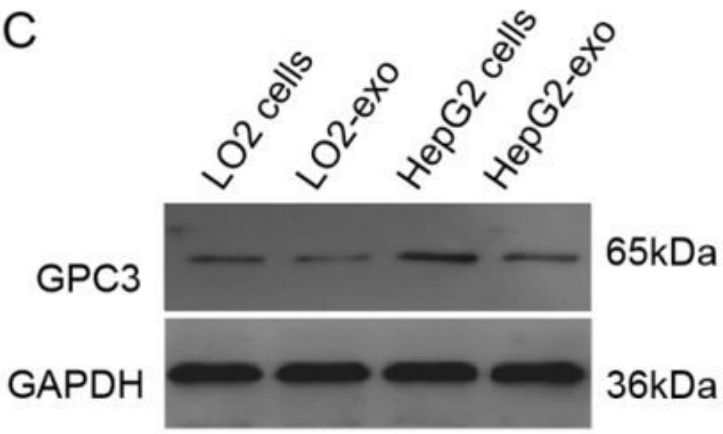

B

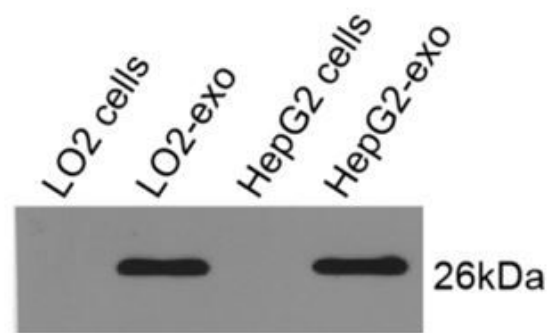

TSG101

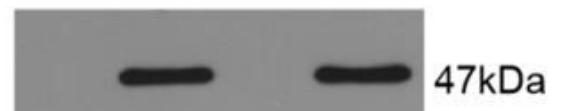

GAPDH

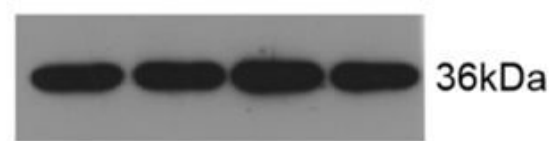

D

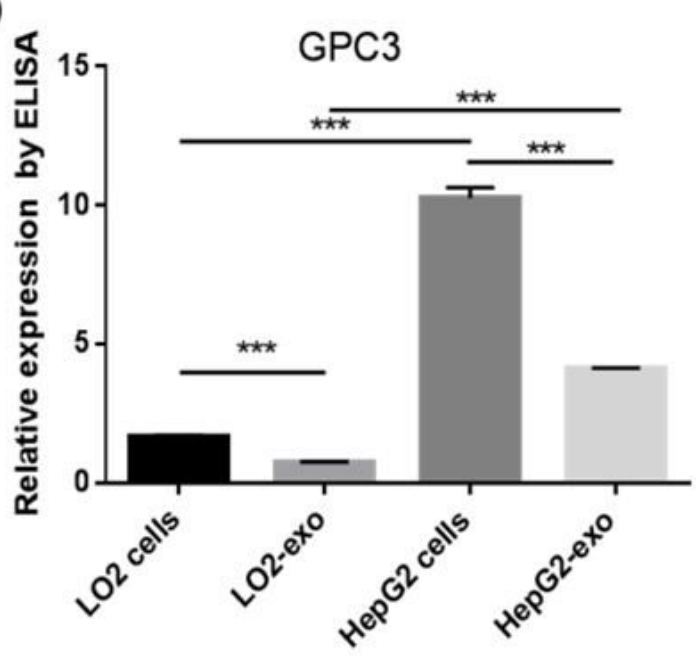

LO2 cells

LO2-exo

HepG2 cells

HepG2-exo

\section{Figure 1}

Characterization of exosomes derived from LO2 cells and HepG2 cells. (A) ZetaView detected the image of exosomes in the separation medium (Scale bars,100 nm). (B) Western blot analysis showed the expression of two exosomal markers of CD63 and TSG101 in the exosome-rich medium. (C) Western blot and (D) ELISA detected the expression of GPC3 in cells and exosomes derived from LO2 and HepG2 cells. $(* \star * P<0.001, n=3)$. 

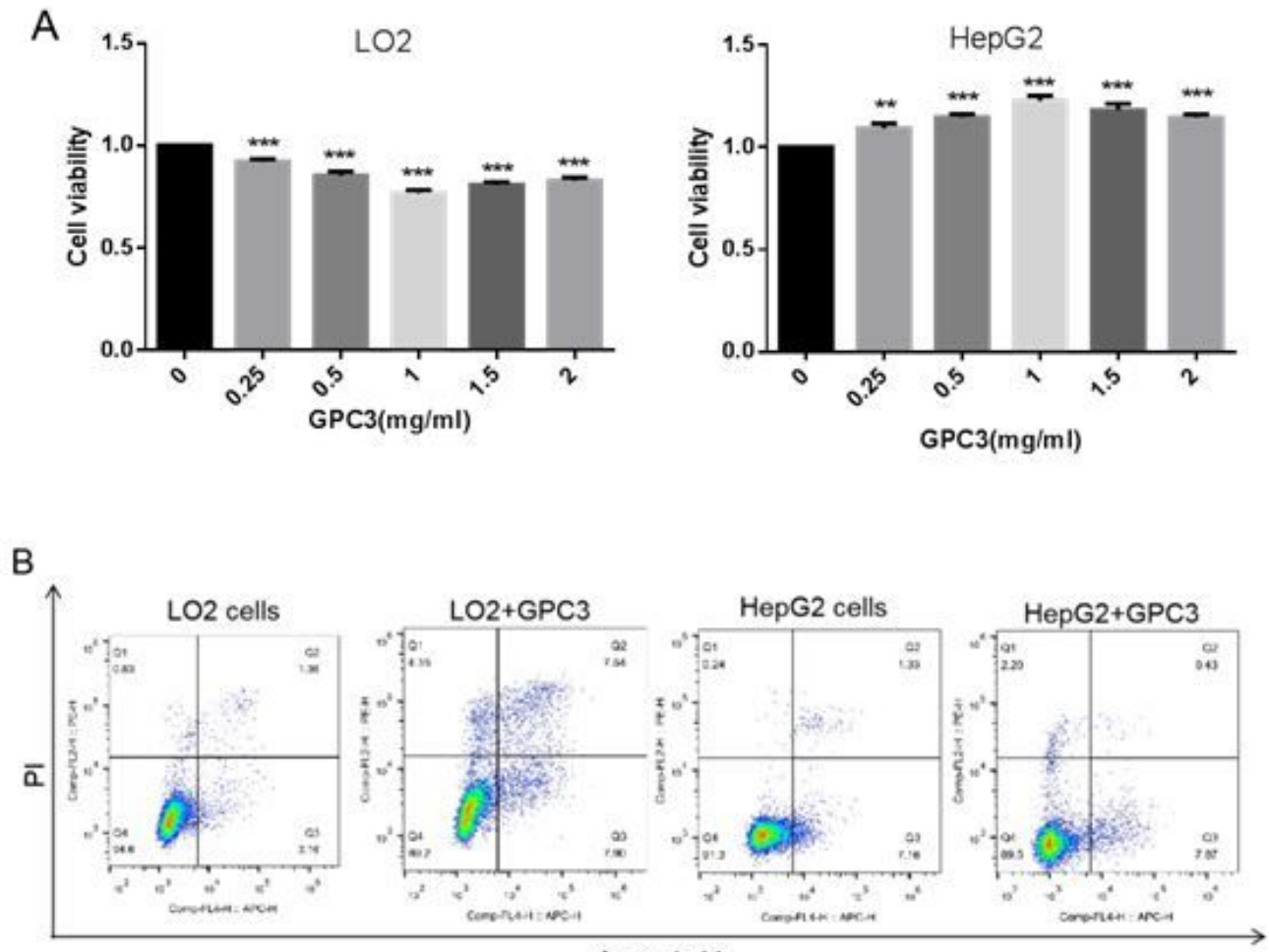

Annexin V

C

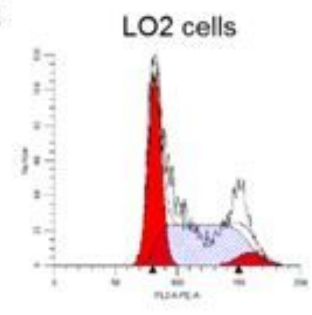

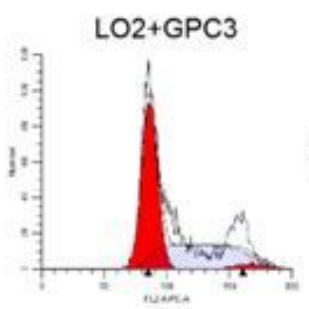
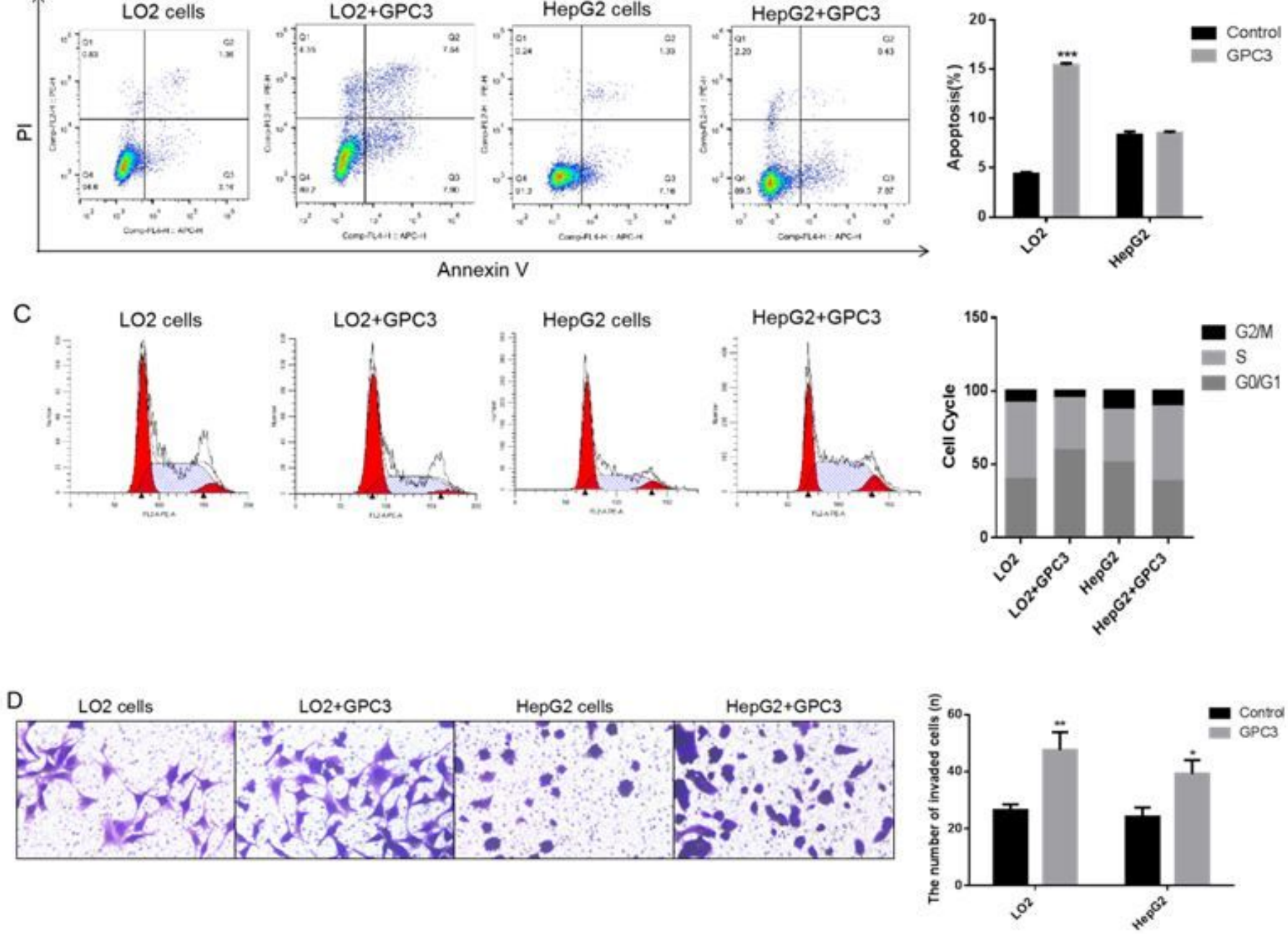

Figure 2

The effect of GPC3 on the proliferation and invasion of HCC cells. (A) The cell proliferation activity of LO2 and HepG2 cells was determined by GPC3 treatment. (B) FITC-Annexin V/PI analysis was performed in LO2 and HepG2 cells treated with GPC3. The FC analysis of Annexin V showed that apoptotic cells increased in LO2 cells treated with GPC3. (C) cell cycle analysis showed that the G0/G1 population increased in LO2 cells treated with GPC3, and decreased in HepG2 cells. (D) Evaluate the invasion ability of cells after 24 hours incubation with GPC3 by Transwell analysis (Scale bar,100 $\mu \mathrm{m}$ ). The data are 


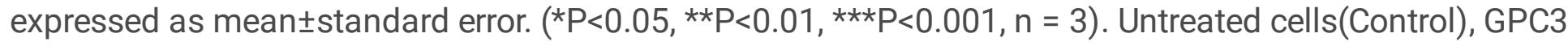
treated LO2 cells, or HepG2 cells (LO2+GPC3/HepG2+GPC3).
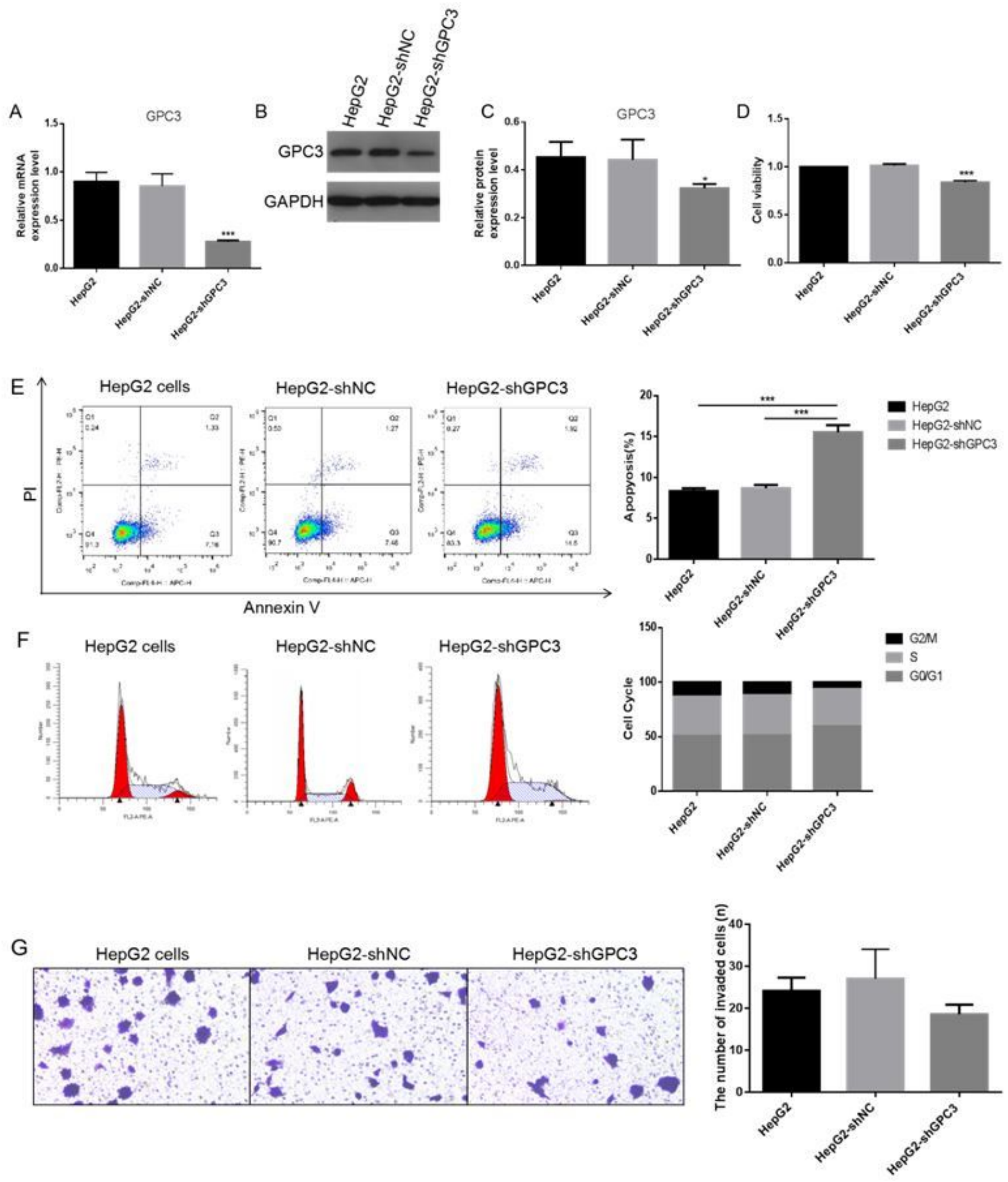

Figure 3

Knockdown of GPC3 inhibited the proliferation and invasion of HCC cells. (A) The level of GPC3 mRNA in HepG2 cells transfected with shRNA was measured by fluorescence quantitative PCR. (B) and (C) were used to determine the GPC3 protein level in HepG2 cells transfected with shRNA by Western blot. (D) 
Determination of cell proliferation viability of HepG2 cells after knocking down GPC3. (E) After knockdown of GPC3, FITC-Annexin V/PI analysis was performed on HepG2 cells. The FC analysis of Annexin V showed that GPC3 knockdown increased apoptotic cells in HepG2 cells. (F) cell cycle analysis showed that knockdown of GPC3 increased the G0/G1 population in HepG2 cells. (G) Evaluate the invasion ability of HepG2 cells after knockdown with GPC3 by Transwell analysis (Scale bar,100 $\mu \mathrm{m}$ ). The data are expressed as mean \pm standard error..ShRNA transfection caused GPC3 knockdown in HepG2 cells (HepG2-shGPC3). ( ${ }^{\star} P<0.05,{ }^{* * P}<0.01,{ }^{* * * P}<0.001$ HepG2 vs HepG2-shGPC3, $\left.n=3\right)$.
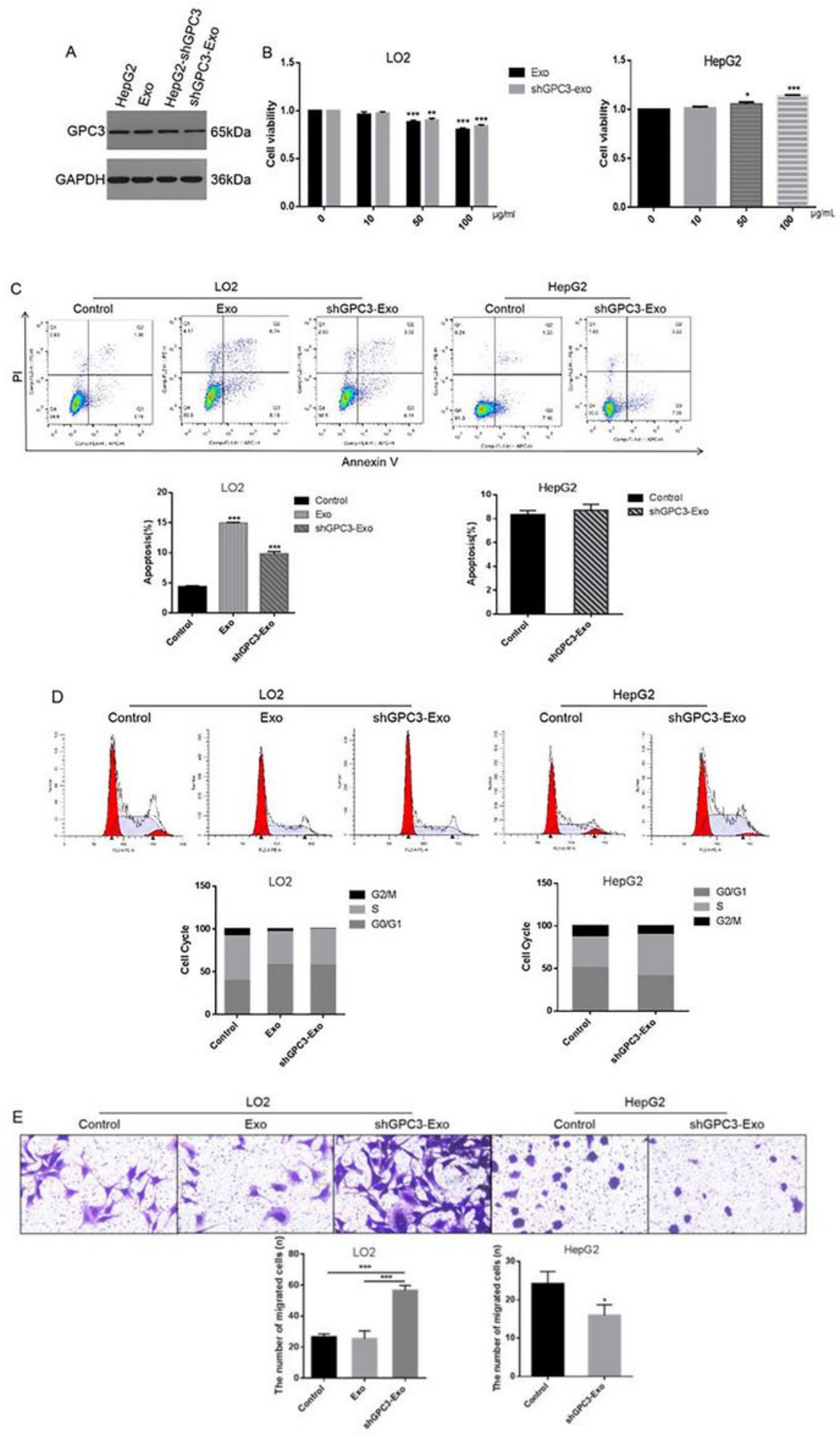


\section{Figure 4}

The effect of GPC3 in liver cancer exosomes on the proliferation and invasion of LO2 and HCC cells. (A) Western blot was used to determine the level of GPC3 protein in HepG2 cells and exosomes with or without GPC3 knockdown. (B) The cell proliferation activity of LO2 and HepG2 cells was determined by treatment with or without GPC3 knockdown HepG2 cell exosomes. ( ${ }^{\star} p<0.05$, ${ }^{\star *} p<0.01$, ${ }^{\star \star \star} p<0.001$ vs 0 , $\mathrm{n}=3$ ). (C) FITC-Annexin V / PI analysis was performed in LO2 and HepG2 cells treated with exosomes. The FC analysis of Annexin V showed that apoptotic cells increased in LO2 cells treated with liver cancer exosomes. (D) Cell cycle analysis showed that the GO/G1 population increased in LO2 cells treated with exosomes, but decreased in HepG2 cells. (E) Assess the invasion ability of cells after 24 hours incubation with exosomes by Transwell analysis (Scale Bar $=100 \mu \mathrm{m}$ ). The data are expressed as mean \pm standard error. ( ${ }^{\star} p<0.05,{ }^{\star \star} p<0.01,{ }^{\star \star \star} \mathrm{p}<0.001$ vs Control $n=3$ ). Untreated cells(Control), HepG2 exosomes treated (Exo) LO2 and HepG2, GPC3 knockdown HepG2 exosomes (shGPC3-Exo) treated LO2 and HepG2. 

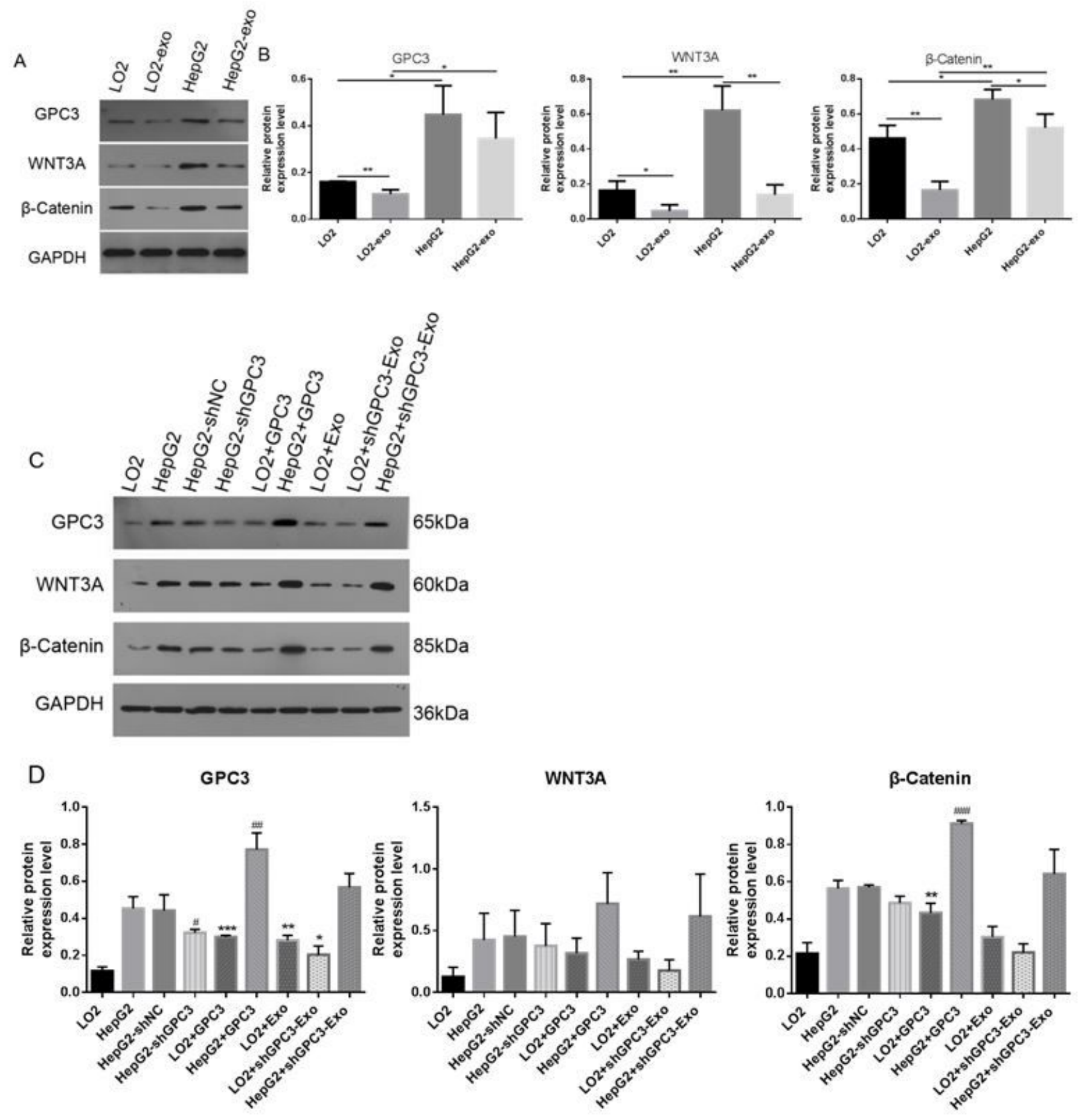

Figure 5

Exosomal GPC3 affects the growth of normal hepatocytes and HCC cells through Wnt/ $\beta$-catenin. (A) and (B) determined the expression level of GPC3 in LO2 and HepG2 cells and exosomes by Western blot analysis. ( ${ }^{\star} p<0.05,{ }^{\star} p<<0.01$, ${ }^{\star *} P<0.001, n=3$ ). (C) and (D), under the influence of GPC3 in different ways, the expression levels of GPC3, WNT3A and $\beta$-catenin in LO2 and HepG2 cells were determined by Western blot analysis. $\left({ }^{\star} p<0.05,{ }^{*} p<0.01,{ }^{\star *} \mathrm{p}<0.001\right.$ vs. LO2; $\# p<0.05, \# \# p<0.01$ vs. HepG2. $\left.n=3\right)$. The data are expressed as mean \pm standard error. 